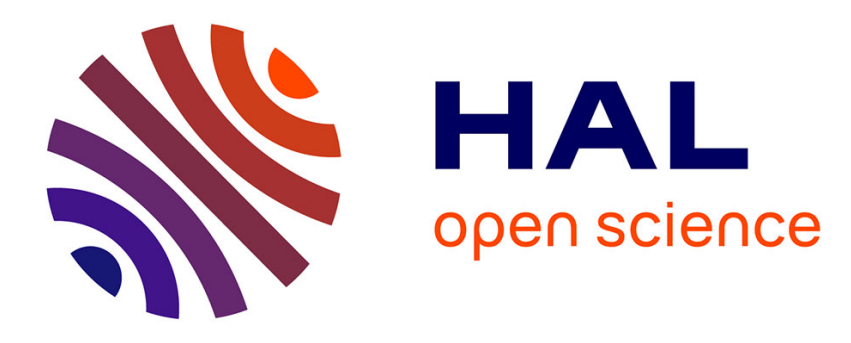

\title{
Consistent collective decisions under majorities based on differences
}

\author{
Mostapha Diss, Patrizia Pérez-Asurmendi
}

\section{To cite this version:}

Mostapha Diss, Patrizia Pérez-Asurmendi. Consistent collective decisions under majorities based on differences. 2014. halshs-00944687

\section{HAL Id: halshs-00944687 https://shs.hal.science/halshs-00944687}

Preprint submitted on 11 Feb 2014

HAL is a multi-disciplinary open access archive for the deposit and dissemination of scientific research documents, whether they are published or not. The documents may come from teaching and research institutions in France or abroad, or from public or private research centers.
L'archive ouverte pluridisciplinaire HAL, est destinée au dépôt et à la diffusion de documents scientifiques de niveau recherche, publiés ou non, émanant des établissements d'enseignement et de recherche français ou étrangers, des laboratoires publics ou privés. 


\section{Consistent collective decisions} under majorities based on differences

Mostapha Diss, Patrizia Pérez-Asurmendi 


\section{GATE Groupe d'Analyse et de Théorie Économique Lyon-St Étienne}

93, chemin des Mouilles 69130 Ecully - France

Tel. +33(0)4 72866060

Fax $+33(0) 472866090$

6, rue Basse des Rives 42023 Saint-Etienne cedex 02 - France

Tel. +33 (0)4 77421960

Fax. +33 (0)4 77421950

Messagerie électronique / Email : gate@gate.cnrs.fr

Téléchargement / Download : http://www.gate.cnrs.fr - Publications / Working Papers 


\title{
Consistent collective decisions under majorities based on differences
}

\author{
Mostapha Diss • \\ Patrizia Pérez-Asurmendi
}

This VERSION: JANUARY 2014

\begin{abstract}
The main criticism to the aggregation of individual preferences under majority rules refers to the possibility of reaching inconsistent collective decisions from the election process. In these cases, the collective preference includes cycles and even could prevent the election of any alternative as the collective choice. The likelihood of consistent outcomes under two classes of majority rules constitutes the aim of this paper. Specifically, we focus on majority rules that require certain consensus in individual preferences to declare an alternative as the winner. In the case of majorities based on difference of votes, such requirement asks to the winner alternative to obtain a difference in votes with respect to the loser alternative taken into account that individuals are endowed with weak preference orderings. Same requirement is asked to the restriction of these rules to individual linear preferences, whereas in the case of majorities based on difference in support, the requirement has to do with the difference in the sum of the intensities for the alternatives in contest.
\end{abstract}

Keywords Majorities based on difference of votes · Majorities based on difference in support · Probability · Transitivity · Triple-acyclicity.

\footnotetext{
M. Diss

Université de Lyon, Lyon, F-69007, France; CNRS, GATE Lyon Saint-Etienne, Ecully, F69130, France; Université Jean Monnet, Saint-Etienne, F-42000, France.

E-mail: diss@gate.cnrs.fr

Corresponding author: P. Pérez-Asurmendi

Grupo de investigación PRESAD, Universidad de Valladolid, Avda. Valle de Esgueva 6 , 47011, Valladolid, Spain

\&

Grupo de investigación SEED, Universidad Pública de Navarra, Campus de Arrosadia, 31006, Pamplona, Spain.

E-mail: patrizia.perez@eco.uva.es
} 


\section{Introduction}

Since Condorcet (1785) introduced the Voting Paradox, it is well known that the aggregation of transitive individual preferences under simple majority rule could lead to inconsistent collective preferences. Recalling the classical example, consider a three-alternative election with alternatives $x_{1}, x_{2}, x_{3}$ and three individuals endowed with the following rankings $x_{1} x_{2} x_{3}, x_{2} x_{3} x_{1}$ and $x_{3} x_{1} x_{2}$, where, for instance, $x_{1} x_{2} x_{3}$ means that $x_{1}$ is preferred to $x_{2}, x_{2}$ is preferred to $x_{3}$ and $x_{1}$ is preferred to $x_{3}$. For each pair of alternatives, each individual casts a vote for her/his preferred alternative following just assumed orderings. Adding up these votes, alternatives $x_{1}, x_{2}$ and $x_{3}$ defeat $x_{2}, x_{3}$ and $x_{1}$ respectively, by two votes to one. In that voting situation, there is a cycle on the ordering induced by the strict collective preference. In such a case, that preference fails on transitivity and on triple-acyclicity given the requirements of such conditions. To illustrate, assume that alternative $x_{1}$ defeats $x_{2}$ and $x_{2}$ defeats $x_{3} ; x_{1}$ defeats $x_{3}$ whenever the strict collective preference is transitive whereas $x_{3}$ does not defeat $x_{1}$ whenever the strict collective preference is triple-acyclic.

Consider now the following voting process' outcome: $x_{1}$ defeats $x_{2}$ and it is indifferent to $x_{3}$, and $x_{2}$ is also indifferent to $x_{3}$. In this case, the weak collective preference fails on consistency. Notice that the strict preference associated with that weak preference behaves right but the indifference relation associated with the weak preference fails on transitivity.

The idea that the Voting Paradox 'certainly could not be very likely to ever be observed in realistic situation' stated by Gehrlein and Fishburn (1976), promotes the probabilistic study of the occurrence of that paradox and of their consequences under different aggregation rules. In several studies, it is assumed an a priori probability model to estimate the likelihood of different voting situations, derived the conditions under which the paradox or the effects of that appear and reached probabilities through combinatoric calculus. In this context, stand out the studies about simple majority rule (Gehrlein and Fishburn 1976; Fishburn and Gehrlein 1980; Gehrlein 1983), supermajority rules (Balasko and Crès 1997; Tovey 1997) or scoring rules (Gehrlein and Fishburn 1980, 1981, 1983; Cervone et al 2005), among others. In other ones, these probabilities are calculated following the Montecarlo simulation methodology. Specifically, the study of the cyclical and intransitive collective decisions under the simple majority rule are carried out in Campbell and Tullock (1965), Klahr (1966), DeMeyer and Plott (1970) and Jones et al (1995).

This paper is devoted to analyze and compare the probabilities of consistent collective decisions over three alternatives for two different classes of majorities rules: majorities based on difference of votes (García-Lapresta and Llamazares 2001; Llamazares 2006; Houy 2007) and majorities based on difference in support (García-Lapresta and Llamazares 2010). Given two alternatives, these majorities based on differences focus on requiring to an alternative, to be declared the winner, to reach a number of votes or a support that exceeds the number of votes or the support for the other alternative in a quantity fixed before the voting process. Therefore, the difference between these two classes 
of majorities restricts to the types of individual preferences considered. In the first case, individual preferences are understood as crisp preferences, i.e. given a pair of alternatives individuals declare if they prefer an alternative to another one or if they are indifferent between them. Here, we distinguish between the case where individuals are endowed with weak preferences and the case where individuals are endowed with linear orderings. In the second case, individual preferences are understood as reciprocal preference relations, i.e. given two alternatives, individuals declare the degree with which they prefer an alternative to another one, in other words their intensities of preference, by means of numerical values in the unit interval.

Coming back to the consistent collective decisions analyzed here, we specifically calculate the probabilities of transitive and triple-acyclic strict collective preferences and the corresponding ones of transitive weak collective preferences for the three specifications of majorities based on differences stated before, as the proportion of collective decisions that fulfill each of such consistency conditions over the total number of possible collective decisions.

To calculate the probabilities of consistent outcomes under majorities based on difference of votes taken into account weak and linear individual preferences respectively, we consider that each individual preference ordering is equiprobable by embracing the Impartial Anonymous Culture (IAC) condition (Gehrlein and Fishburn 1976) to describe the likelihood of the possible individual orderings. On the one hand, assuming weak or linear individual orderings jointly with the IAC condition allow to know the total number of possible collective preferences (again, Gehrlein and Fishburn 1976). On the other hand, the number of consistent profiles is calculated by means of Ehrhart polynomials, a method recently introduced in the social choice literature by Wilson and Pritchard (2007) and Lepelley et al (2008) in order to estimate the probabilities of some voting paradoxes under the IAC condition.

In the case of the calculations of the probabilities of consistent outcomes under majorities based on difference in support, we propose to apply the Montecarlo simulation methodology to estimate such probabilities inspired by the studies in Campbell and Tullock (1965), Klahr (1966), DeMeyer and Plott (1970) and Jones et al (1995).

Specifically, we generate the individual reciprocal preference relations for the case of three alternatives. Each individual intensity of preference is understood as a continuous random variable in the unit interval consistently built with a specific transitivity condition over the individual's reciprocal preference relations. Then, we fix the required difference in support and aggregate these individual preferences with the corresponding majority based on difference in support. We derive the resultant collective ordering of alternatives and evaluate its consistency. Finally, we iterate that procedure to estimate desired probabilities as the number of consistent orderings over the total number of simulated collective orderings.

The methodology proposed here allows us to hypothesize about a relationship between the type of individual preferences assumed under each rule and the likelihood of inconsistent collective decisions. Moreover, we set forth our 
results for majorities based on difference of votes with previous ones on simple majority (Gehrlein (1997) and Lepelley and Martin (2001)). We also are able to analyze the impact of the types of transitivity conditions for individual reciprocal preferences on the probability of consistent collective decisions under majorities based on difference in support. In addition, we compare our results on probabilities with some theoretical ones about the consistency of the collective preferences under majorities based on difference in support (Llamazares et al 2013; Llamazares and Pérez-Asurmendi 2013).

The paper is organized as follows. Section 2 describes the theoretical framework followed in this paper and introduces majorities based on difference of votes and majorities based on difference in support. Sections 3 and 4 provide the results about the probability of consistent collective decisions under majorities based on difference of votes with linear preferences and with weak preferences, respectively. Section 5 is devoted to the simulated probabilities in the case of majorities based on difference in support. Section 6 discusses the results and concludes.

\section{Preliminaries}

Consider a set of alternatives $X=\left\{x_{1}, x_{2}, x_{3}\right\}$ in an election with $m$ individuals. Let $S$ be a binary relation on $X$, i.e. a subset of the cartesian product $X \times X$. In what follows, $x_{i} S x_{j}$ stands for $\left(x_{i}, x_{j}\right) \in S$, i.e. when $x_{i}$ is in the relation $S$ with $x_{j} . S^{-1}$ is the inverse relation of $S$ defined by $x_{i} S^{-1} x_{j} \Leftrightarrow x_{j} S x_{j}$ and $S^{c}$ is the complement relation of $S$ defined by $x_{i} S^{c} x_{j} \Leftrightarrow \neg\left(x_{i} S x_{j}\right)$. Given two binary relations $S$ and $T$, the intersection of $S$ and $T$ is also a binary relation defined by $x_{i}(S \cap T) x_{j} \Leftrightarrow\left(x_{i} S x_{j} \wedge x_{i} T x_{j}\right)$. A binary relation $S$ on $X$ is

1. reflexive if $\forall x \in X, x S x$,

2. symmetric if $\forall x_{i}, x_{j} \in X, x_{i} S x_{j} \Rightarrow x_{j} S x_{i}$,

3. asymmetric if $\forall x_{i}, x_{j} \in X, x_{i} S x_{j} \Rightarrow \neg\left(x_{j} S x_{i}\right)$,

4. antisymmetric if $\forall x_{i}, x_{j} \in X,\left(x_{i} S x_{j} \wedge x_{j} S x_{i}\right) \Rightarrow x_{i}=x_{j}$,

5. complete if $\forall x_{i}, x_{j} \in X, x_{i} S x_{j} \vee x_{j} S x_{i}$,

6. transitive if $\forall x_{i}, x_{j}, x_{l} \in X,\left(x_{i} S x_{j} \wedge x_{j} S x_{l}\right) \Rightarrow x_{i} S x_{l}$,

7. triple-acyclic if $\forall x_{i}, x_{j}, x_{l} \in X,\left(x_{i} S x_{j} \wedge x_{j} S x_{l}\right) \Rightarrow \neg\left(x_{l} S x_{i}\right)$.

A weak preference $R$ is a complete binary relation on the set of alternatives $X$. The strict preference $P$ associated with $R$ is the asymmetric binary relation on $X$ defined by $P=\left(R^{-1}\right)^{c}$ and the corresponding indifference relation $I$ is the reflexive and symmetric binary relation on $X$ defined by $I=R \cap R^{-1}$. $\mathcal{P}(X)$ is the set of strict preferences. A weak ordering is a transitive weak preference whereas a linear ordering is also antisymmetric.

From definitions above it is well know that any weak ordering implies a transitive strict preference relation and a transitive indifference relation. Moreover, any transitive strict preference is also a triple-acyclic preference relation. Notice that the converse is not true. 
Given that the social decision between two alternatives is given by either a strict preference relation or an indifference relation, and that three alternatives are in contest, we consider the 27 cases in Table 1 as possible social outcomes.

Table 1 Possible social outcomes in a three alternative election.

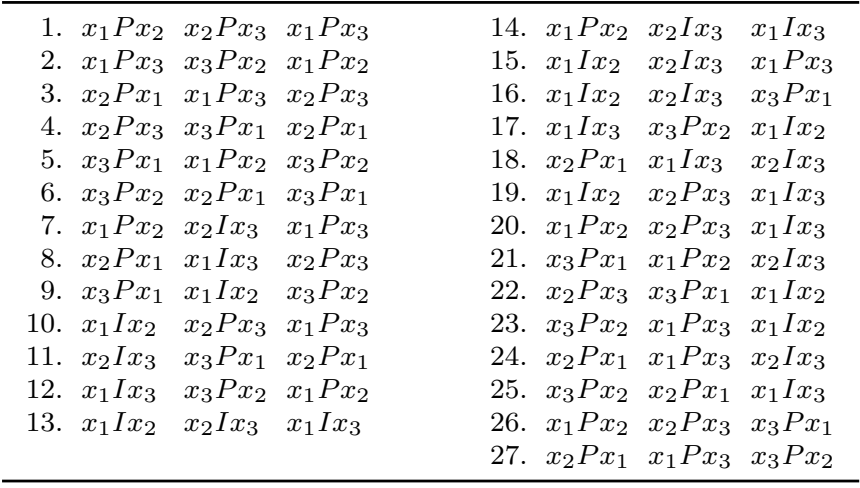

Our interest focuses on the frequency of consistent social outcomes given the 27 possible outcomes above. We distinguish among three cases of consistent outcomes; the case of weak orderings corresponding to the first thirteen outcomes, the case of transitive strict preferences corresponding to the first nineteen and the case of triple-acyclic strict preferences corresponding to the first twenty-fifth outcomes.

\subsection{Individual preferences}

We consider that individuals compare the alternatives on $X$ by pairs and declare their preferences by means of values $r_{i j}^{p} \in[0,1]$, where $r_{i j}^{p}>0.5$ means that alternative $x_{i}$ is somewhat preferred to $x_{j}$ by the individual $p$, whereas $r_{i j}^{p}<0.5$ signifies that alternative $x_{j}$ is somewhat preferred to $x_{i}$ by the individual $p$. At that point, we distinguish between the general case in which preferences are represented by reciprocal preferences and the particular case of that, referred to as crisp preferences.

1. Crisp preferences: the values of $r_{i j}^{p}$ are restricted to the set of discrete values $\{0,0.5,1\}$. If $r_{i j}^{p}=1$, individual $p$ prefers alternative $x_{i}$ to alternative $x_{j}$, whereas if $r_{i j}^{p}=0$, individual $p$ prefers $x_{j}$ to $x_{i}$. If $r_{i j}^{p}=0.5$, individual $p$ is indifferent between both alternatives. Condition $r_{i j}^{p}+r_{j i}^{p}=1$ guarantees that the preference of individual $p$ is a weak preference. Moreover, the conditions

$$
\begin{gathered}
\left(r_{i j}^{p}=1 \wedge r_{j l}^{p}=1\right) \Rightarrow r_{i l}^{p}=1, \\
\left(r_{i j}^{p}=0.5 \wedge r_{j l}^{p}=0.5\right) \Rightarrow r_{i l}^{p}=0.5,
\end{gathered}
$$


assure that the preference of individual $p$ is a weak ordering. Individual linear orderings could also be represented in this framework by including the following condition: $r_{i j}^{p} \in\{0,1\} \forall i \neq j$. Thus, individuals could only be indifferent between an alternative and itself.

2. Reciprocal preferences: the values of $r_{i j}^{p}$ belong to the unit interval $[0,1]$ with the following interpretation: $r_{i j}^{p}>0.5$ indicates that the individual $p$ prefers the alternative $x_{i}$ to the alternative $x_{j}$, the more the nearer is the value of $r_{i j}^{p}$ to 1 that represents the maximum degree of preference for $x_{i}$ over $x_{j}$; conversely, $r_{i j}^{p}<0.5$, means that individual $p$ prefers alternative $x_{j}$ to $x_{i}$, the more the nearer is the value of $r_{i j}^{p}$ to 0 that represents the maximum degree of preference for $x_{j}$ over $x_{i}$; finally, $r_{i j}^{p}=0.5$ stands for the indifference between $x_{i}$ and $x_{j}$ for individual $p$. The reciprocity of these preferences is described by the condition $r_{i j}^{p}+r_{j i}^{p}=1$. To avoid the possibility of having incoherent individual preferences, we need to assume some kind of rationality condition. But, in this framework, several concepts could be taken to ensure such rationality requirement (see, among others, Zadeh 1971; Dubois and Prade 1980; Dasgupta and Deb 1996; GarcíaLapresta and Meneses 2005). Here, we consider the following transitivity conditions for reciprocal preference relations.

Definition 1 We say that individual $p$ is

(a) 0.5 -transitive if $\forall i, j, l \in\{1,2,3\}$

$$
\left(r_{i j}^{p}>0.5 \wedge r_{j l}^{p}>0.5\right) \Rightarrow r_{i l}^{p}>0.5
$$

(b) min-transitive if $\forall i, j, l \in\{1,2,3\}$

$$
\left(r_{i j}^{p}>0.5 \wedge r_{j l}^{p}>0.5\right) \Rightarrow r_{i l}^{p} \geq \min \left\{r_{i j}^{p}, r_{j l}^{p}\right\}
$$

(c) am-transitive if $\forall i, j, l \in\{1,2,3\}$

$$
\left(r_{i j}^{p}>0.5 \wedge r_{j l}^{p}>0.5\right) \Rightarrow r_{i l}^{p} \geq\left(r_{i j}^{p}+r_{j l}^{p}\right) / 2,
$$

(d) max-transitive if $\forall i, j, l \in\{1,2,3\}$

$$
\left(r_{i j}^{p}>0.5 \wedge r_{j l}^{p}>0.5\right) \Rightarrow r_{i l}^{p} \geq \max \left\{r_{i j}^{p}, r_{j l}^{p}\right\} \text {. }
$$

The preferences of each individual over the alternatives in $X=\left\{x_{1}, x_{2}, x_{3}\right\}$ can be represented using a $3 \times 3$ matrix $R^{p}=\left(r_{i j}^{p}\right)$ as follows:

$$
R^{p}=\left(\begin{array}{ccc}
0.5 & r_{12}^{p} & r_{13}^{p} \\
1-r_{12}^{p} & 0.5 & r_{23}^{p} \\
1-r_{13}^{p} & 1-r_{23}^{p} & 0.5
\end{array}\right)
$$

Individual preferences are collected in a vector where each vector-element represents the preferences of an individual. Assuming $m$ individuals ${ }^{1}$ and taking into account the above distinction among linear, weak and reciprocal preferences, a profile of linear orderings is a vector $\left(R^{1}, \ldots, R^{m}\right) \in \mathcal{L}(X)^{m}$, being

1 To calculate the probabilities presented here, $m$ takes the following values: $3,4,5,10$, $100,1,000$ and 100,000 . 
$\mathcal{L}(X)$ the set of all linear orderings; a profile of weak orderings is a vector $\left(R^{1}, \ldots, R^{m}\right) \in \mathcal{W}(X)^{m}$, being $\mathcal{W}(X)$ the set of all weak orderings; and a profile of reciprocal preferences is a vector $\left(R^{1}, \ldots, R^{m}\right) \in \mathcal{R}(X)^{m}$, being $\mathcal{R}(X)$ the set of all reciprocal preference relations.

\subsection{Majorities based on differences}

Given the nature of the three types of individual preferences examined in Subsection 2.1, we also consider three different specifications of majorities based on differences relying on the types of individual preferences that we take into account in each case. In what follows, we refer to these majorities as majorities based on difference of votes with linear orderings, majorities based on difference of votes with weak orderings and majorities based on difference in support for reciprocal preference relations.

The concept of majorities based on difference of votes was introduced in García-Lapresta and Llamazares (2001) and was later axiomatically characterized in Llamazares (2006), and subsequently in Houy (2007). These rules involve crisp preferences, i.e. given a pair of alternatives, individuals could declare their preference for one of them or their indifference between both alternatives.

Under these majorities, an alternative, say $x_{i}$, is declared the winner if the number of individuals who prefer that alternative, to the other one, say $x_{j}$, exceeds the number of individuals who prefer $x_{j}$ to $x_{i}$ in a difference of votes, fixed before the election process. Assuming $m$ individuals, that difference could take any integer value in $\{0, \ldots, m-1\}$. These majorities are located between simple majority rule where the difference of votes is zero and unanimity where the difference of votes is the total number of individuals $m$ minus one. Moreover, if the indifference state is ruled out from individual preferences, these majorities are equivalent to supermajority rules.

Taking into account the former majorities based on difference of votes, we introduce in Definition 2 the majorities based on difference of votes with linear orderings and in Definition 3 the majorities based on difference of votes with weak orderings. In what follows, the symbol \# stands for the cardinality of a set. 
Definition 2 (Majorities based on difference of votes with linear orderings or $M_{k^{\prime}}^{L}$ majorities)

Given $k^{\prime} \in\{0,1, \ldots, m-1\}$, the majority based on difference of votes with linear orderings or $M_{k^{\prime}}^{L}$ majority is the mapping $M_{k^{\prime}}^{L}: \mathcal{L}(X)^{m} \longrightarrow \mathcal{P}(X)$ defined by $M_{k^{\prime}}^{L}\left(R^{1}, \ldots, R^{m}\right)=P_{k^{\prime}}^{L}$, where

$$
x_{i} P_{k^{\prime}}^{L} x_{j} \Leftrightarrow \#\left\{p \mid r_{i j}^{p}=1\right\}>\#\left\{p \mid r_{j i}^{p}=1\right\}+k^{\prime} .
$$

The indifference relation associated with $P_{k^{\prime}}^{L}$ is as follows:

$$
x_{i} I_{k^{\prime}}^{L} x_{j} \Leftrightarrow\left|\#\left\{p \mid r_{i j}^{p}=1\right\}-\#\left\{p \mid r_{j i}^{p}=1\right\}\right| \leq k^{\prime} .
$$

Example 1 Let $R^{I}$ and $R^{I I}$ be the following individual linear preference orderings over the alternatives on $X=\left\{x_{1}, x_{2}, x_{3}\right\}$.

$$
R^{I}=\left(\begin{array}{ccc}
0.5 & 1 & 1 \\
0 & 0.5 & 1 \\
0 & 0 & 0.5
\end{array}\right), \quad R^{I I}=\left(\begin{array}{ccc}
0.5 & 0 & 1 \\
1 & 0.5 & 1 \\
0 & 0 & 0.5
\end{array}\right)
$$

Consider the profile $\left(R^{1}, R^{2}, R^{3}, R^{4}, R^{5}\right)$ where

$$
R^{p}= \begin{cases}R^{I} & \text { if } p=1,2,3, \\ R^{I I} & \text { if } p=4,5 .\end{cases}
$$

Assuming a required difference of votes $k^{\prime}$ equal to 1 and applying the corresponding $M_{1}^{L}$ majority we have

$$
\begin{gathered}
\left|\#\left\{p \mid r_{12}^{p}=1\right\}-\#\left\{p \mid r_{21}^{p}=1\right\}\right|=|3-2| \leq 1 \Rightarrow x_{1} I_{1}^{L} x_{2}, \\
\#\left\{p \mid r_{23}^{p}=1\right\}=5>\#\left\{p \mid r_{32}^{p}=1\right\}+1=0+1 \Rightarrow x_{2} P_{1}^{L} x_{3}, \\
\#\left\{p \mid r_{13}^{p}=1\right\}=5>\#\left\{p \mid r_{31}^{p}=1\right\}+1=0+1 \Rightarrow x_{1} P_{1}^{L} x_{3} .
\end{gathered}
$$

Definition 3 (Majorities based on difference of votes with weak orderings or $M_{k^{\prime}}$ majorities)

Given $k^{\prime} \in\{0, \ldots, m-1\}$, the majority based on difference of votes with weak orderings or $M_{k^{\prime}}$ majority is the mapping $M_{k^{\prime}}: \mathcal{W}(X)^{m} \longrightarrow \mathcal{P}(X)$ defined by $M_{k^{\prime}}\left(R^{1}, \ldots, R^{m}\right)=P_{k^{\prime}}$, where

$$
x_{i} P_{k^{\prime}} x_{j} \Leftrightarrow \#\left\{p \mid r_{i j}^{p}=1\right\}>\#\left\{p \mid r_{j i}^{p}=1\right\}+k^{\prime} .
$$

The indifference relation associated with $P_{k^{\prime}}$ is as follows:

$$
x_{i} I_{k^{\prime}} x_{j} \Leftrightarrow\left|\#\left\{p \mid r_{i j}^{p}=1\right\}-\#\left\{p \mid r_{j i}^{p}=1\right\}\right| \leq k^{\prime} .
$$


Example 2 Let $R^{I}$ and $R^{I I}$ be the following individual weak preference orderings over the alternatives on $X=\left\{x_{1}, x_{2}, x_{3}\right\}$.

$$
R^{I}=\left(\begin{array}{ccc}
0.5 & 1 & 0.5 \\
0 & 0.5 & 0 \\
0.5 & 1 & 0.5
\end{array}\right), \quad R^{I I}=\left(\begin{array}{ccc}
0.5 & 0.5 & 1 \\
0.5 & 0.5 & 1 \\
0 & 0 & 0.5
\end{array}\right)
$$

Consider the profile $\left(R^{1}, R^{2}, R^{3}, R^{4}, R^{5}\right)$ where

$$
R^{p}= \begin{cases}R^{I} & \text { if } p=1,2,3 \\ R^{I I} & \text { if } p=4,5\end{cases}
$$

Assuming a required difference of votes $k^{\prime}$ equal to 2 and applying the corresponding $M_{2}$ majority we have

$$
\begin{gathered}
\#\left\{p \mid r_{12}^{p}=1\right\}=3>\#\left\{p \mid r_{21}^{p}=1\right\}+2=0+2 \Rightarrow x_{1} P_{2} x_{2} \\
\left|\#\left\{p \mid r_{23}^{p}=1\right\}-\#\left\{p \mid r_{32}^{p}=1\right\}\right|=|2-3| \leq 2 \Rightarrow x_{2} I_{2} x_{3} \\
\left|\#\left\{p \mid r_{13}^{p}=1\right\}-\#\left\{p \mid r_{31}^{p}=1\right\}\right|=|2-0| \leq 2 \Rightarrow x_{1} I_{2} x_{3}
\end{gathered}
$$

In García-Lapresta and Llamazares (2010), majorities based on differences of votes were extended to the framework of reciprocal preferences allowing individuals to declare their degrees of preferences over pairs of alternatives. Majorities based on difference in support allow us to aggregate each profile of reciprocal preferences into a strict collective preference $P_{k}$ over the set of alternatives. Under these rules, the winner alternative is required to reach a support that exceeds the support for the other alternative in a quantity, fixed before the voting process. Formal definition for these majorities is as follows.

Definition 4 (Majorities based on difference in support or $\widetilde{M}_{k}$ majorities (García-Lapresta and Llamazares 2010))

Given $k \in[0, m)$, the majority based on difference in support or $\widetilde{M}_{k}$ majority is the mapping $\widetilde{M}_{k}: \mathcal{R}(X)^{m} \longrightarrow \mathcal{P}(X)$ defined by $\widetilde{M}_{k}\left(R^{1}, \ldots, R^{m}\right)=P_{k}$, where

$$
x_{i} P_{k} x_{j} \Leftrightarrow \sum_{p=1}^{m} r_{i j}^{p}>\sum_{p=1}^{m} r_{j i}^{p}+k
$$

The indifference relation associated with $P_{k}$ is defined by:

$$
x_{i} I_{k} x_{j} \Leftrightarrow\left|\sum_{p=1}^{m} r_{i j}^{p}-\sum_{p=1}^{m} r_{j i}^{p}\right| \leq k .
$$

Notice that when considering crisp preferences, the expression in (2) goes for defining the majorities based on difference of votes with linear and weak preference orderings. 
Example 3 Let $R^{I}$ and $R^{I I}$ be the following reciprocal preference relations over the alternatives on $X=\left\{x_{1}, x_{2}, x_{3}\right\}$.

$$
R^{I}=\left(\begin{array}{ccc}
0.5 & 1 & 0.9 \\
0 & 0.5 & 0.6 \\
0.1 & 0.4 & 0.5
\end{array}\right), \quad R^{I I}=\left(\begin{array}{ccc}
0.5 & 0.8 & 1 \\
0.2 & 0.5 & 0.7 \\
0 & 0.3 & 0.5
\end{array}\right)
$$

Consider the profile $\left(R^{1}, R^{2}, R^{3}, R^{4}, R^{5}\right)$ where

$$
R^{p}= \begin{cases}R^{I} & \text { if } p=1,2,3, \\ R^{I I} & \text { if } p=4,5 .\end{cases}
$$

Assuming a required difference in support $k$ equal to 1.75 and applying the corresponding $\widetilde{M}_{1.75}$ majority we have

$$
\begin{gathered}
\sum_{p=1}^{5} r_{12}^{p}=4.6>\sum_{p=1}^{5} r_{21}^{p}+1.75=0.4+1.75 \Rightarrow x_{1} P_{1.75} x_{2}, \\
\left|\sum_{p=1}^{5} r_{23}^{p}-\sum_{p=1}^{5} r_{32}^{p}\right|=|3.2-1.8| \leq 1.75 \Rightarrow x_{2} I_{1.75} x_{3}, \\
\sum_{p=1}^{5} r_{13}^{p}=4.7>\sum_{p=1}^{5} r_{31}^{p}+1.75=0.3+1.75 \Rightarrow x_{1} P_{1.75} x_{3} .
\end{gathered}
$$

\section{Probability of consistent collective decisions under majorities based on difference of votes with linear orderings}

In this section the results about the probabilities of consistent collective decisions under $M_{k^{\prime}}^{L}$ majorities are introduced under IAC assumption. Given that voters are endowed with complete linear preference orderings, there are six possible preference orders that they might have,

$$
\begin{array}{lll}
x_{1} x_{2} x_{3}\left(m_{1}\right) & x_{1} x_{3} x_{2}\left(m_{2}\right) & x_{2} x_{1} x_{3}\left(m_{3}\right) \\
x_{2} x_{3} x_{1}\left(m_{4}\right) & x_{3} x_{1} x_{2}\left(m_{5}\right) & x_{3} x_{2} x_{1}\left(m_{6}\right)
\end{array}
$$

where $m_{i}$ is the number of voters with the associated linear preference ordering. In this framework, a voting situation is a vector $\boldsymbol{m}=\left(m_{1}, m_{2}, m_{3}, m_{4}, m_{5}, m_{6}\right)$ such that $\sum_{i=1}^{6} m_{i}=m$. As the IAC condition is assumed, all possible voting situations $\boldsymbol{m}$ are equally liked to be observed. Gehrlein and Fishburn (1976) showed that for $m$ agents and 3 alternatives, the total number of voting situations $\boldsymbol{m}$ is given by the expression:

$$
\psi(m)=\frac{(m+1)(m+2)(m+3)(m+4)(m+5)}{120} .
$$


3.1 Probabilities of triple-acyclic strict preferences under majorities based on difference of votes with linear orderings

To calculate the probability of triple-acyclic strict preferences under $M_{k^{\prime}}^{L}$ majorities we focus on the cases from 1 to 25 in Table 1 . Specifically, we first calculate the probability of cyclic strict preferences, i.e. the probability of having preferences like the ones described in the cases 26 and 27 (see again Table 1). Thereafter, we obtain the probability of triple-acyclic cases as 1 minus the probability of cyclic strict preferences.

Going deeper on the strict preference described in the case 26, we notice that for such preference to exist, the numbers of voters associated with the linear orderings described in (4) have to fulfil the following conditions: $m_{1}+$ $m_{2}-m_{3}-m_{4}+m_{5}-m_{6}>k^{\prime}, m_{1}-m_{2}+m_{3}+m_{4}-m_{5}-m_{6}>k^{\prime}$ and $-m_{1}-m_{2}-m_{3}+m_{4}+m_{5}+m_{6}>k^{\prime}$.

In other words, the strict preference in the case 26 requires a voting situation $\boldsymbol{m}=\left(m_{1}, m_{2}, m_{3}, m_{4}, m_{5}, m_{6}\right)$ that fulfils the conditions given by the system of inequalities below.

$$
\left(x_{1} P_{k^{\prime}}^{L} x_{2}, x_{2} P_{k^{\prime}}^{L} x_{3} \text { and } x_{3} P_{k^{\prime}}^{L} x_{1}\right) \Rightarrow\left\{\begin{array}{l}
m_{1}+m_{2}-m_{3}-m_{4}+m_{5}-m_{6}>k^{\prime}, \\
m_{1}-m_{2}+m_{3}+m_{4}-m_{5}-m_{6}>k^{\prime}, \\
-m_{1}-m_{2}-m_{3}+m_{4}+m_{5}+m_{6}>k^{\prime}, \\
m_{i} \geq 0 \text { for } i \in\{1, \ldots, 6\}, \\
m-1 \geq k^{\prime} \geq 0, \\
m_{1}+m_{2}+m_{3}+m_{4}+m_{5}+m_{6}=m .
\end{array}\right.
$$

Therefore, to calculate the probability of cyclic strict preferences, we need to solve the system of linear inequalities derived from conditions that the numbers of voters associated with the linear orderings in (4) have to hold for strict preferences like the ones in the cases 26 and 27 to exist.

We compute the number of voting situations that fulfil these conditions by means of the Parameterized Barvinok's algorithm (Verdoolaege et al (2004)) ${ }^{2}$. Such algorithm allows to quantify the number of integer solutions for systems of inequalities with parameters. The connection of such algorithm to Social Choice Theory was recently pointed out by Wilson and Pritchard (2007) and Lepelley et al (2008).

Given the two parameters $m$ and $k^{\prime}$, the number of voting situations $\boldsymbol{m}$ for our system is given by bivariate quasi polynomials in $m$ and $k^{\prime}$ with 2 periodic coefficients meaning that such coefficients depend on the parity of the parameters $m$ and $k^{\prime}$. Following the notation introduced in Lepelley et al (2008), we represent these coefficients by a list of 2 rational numbers enclosed in square brackets. To illustrate, assume the bracketed list $\left[[a, b]_{m},[b, a]_{m}\right]_{k^{\prime}}$. In the case of even $k^{\prime}$, the relevant list corresponds to $[a, b]_{m}$. The coefficient will be either $a$ when $m$ is even or $b$ when $m$ is odd. Accordingly, in the case

2 The free software to calculate the integer points under the Parameterized Barvinok's algorithm can be found in http://freecode.com/projects/barvinok. 
of odd $k^{\prime}$, the relevant list is $[b, a]_{m}$ and therefore, the coefficient will be either $b$ when $m$ is even or $a$ when $m$ is odd. Thus, the coefficient will be $a$ when $m$ and $k^{\prime}$ have the same the parity and $b$ otherwise.

Notice that in the case of the cyclical strict preferences depicted in the cases 26 and 27 (Table 1), the number of solutions in the system of inequalities derived from the strict preference in the case 26 is the same as in the system derived from the strict preference in the case 27 given the symmetry of such cases. The program indicates that the corresponding quasi polynomial for each of these cases is as follows:

$$
\begin{aligned}
& -\frac{81}{1280} k^{\prime 5}+\left(\frac{27}{256} m+\left[\left[0, \frac{81}{256}\right]_{m},\left[\frac{81}{256}, 0\right]_{m}\right]_{k^{\prime}}\right) k^{\prime 4}+ \\
& \left(-\frac{9}{128} m^{2}+\left[\left[0,-\frac{27}{64}\right]_{m},\left[-\frac{27}{64}, 0\right]_{m}\right]_{k^{\prime}} m+\right. \\
& \left.\left[\left[\frac{9}{64},-\frac{63}{128}\right]_{m},\left[-\frac{63}{128}, \frac{9}{64}\right]_{m}\right]_{k^{\prime}}\right) k^{\prime 3}+ \\
& \left(\frac{3}{128} m^{3}+\left[\left[0, \frac{27}{128}\right]_{m},\left[\frac{27}{128}, 0\right]_{m}\right]_{k^{\prime}} m^{2}+\right. \\
& \left.\left[\left[-\frac{9}{64}, \frac{63}{128}\right]_{m},\left[\frac{63}{128},-\frac{9}{64}\right]_{m}\right]_{k^{\prime}} m+\left[\left[0, \frac{27}{128}\right]_{m},\left[\frac{27}{128}, 0\right]_{m}\right]_{k^{\prime}}\right) k^{\prime 2}+ \\
& \left(-\frac{1}{256} m^{4}+\left[\left[0,-\frac{3}{64}\right]_{m},\left[-\frac{3}{64}, 0\right]_{m}\right]_{k^{\prime}} m^{3}+\right. \\
& {\left[\left[\frac{3}{64},-\frac{21}{128}\right]_{m},\left[-\frac{21}{128}, \frac{3}{64}\right]_{m}\right]_{k^{\prime}} m^{2}+\left[\left[0,-\frac{9}{64}\right]_{m},\left[-\frac{9}{64}, 0\right]_{m}\right]_{k^{\prime}} m+} \\
& \left.\left[\left[-\frac{1}{20}, \frac{71}{1280}\right]_{m},\left[\frac{71}{1280},-\frac{1}{20}\right]_{m}\right]_{k^{\prime}}\right) k^{\prime}+ \\
& \frac{1}{3840} m^{5}+\left[\left[0, \frac{1}{256}\right]_{m},\left[\frac{1}{256}, 0\right]_{m}\right]_{k^{\prime}} m^{4}+ \\
& {\left[\left[-\frac{1}{192}, \frac{7}{384}\right]_{m},\left[\frac{7}{384},-\frac{1}{192}\right]_{m}\right]_{k^{\prime}} m^{3}+} \\
& {\left[\left[0, \frac{3}{128}\right]_{m},\left[\frac{3}{128}, 0\right]_{m}\right]_{k^{\prime}} m^{2}+\left[\left[\frac{1}{60},-\frac{71}{3840}\right]_{m},\left[-\frac{71}{3840}, \frac{1}{60}\right]_{m}\right]_{k^{\prime}} m+} \\
& {\left[\left[0,-\frac{7}{256}\right]_{m},\left[-\frac{7}{256}, 0\right]_{m}\right]_{k^{\prime}} \text {. }}
\end{aligned}
$$

In addition, the program points out that this relation holds only if $k^{\prime} \leq$ $(m-3) / 3$. Otherwise, the number of voting situations is zero. 
We simplify ${ }^{3}$ the quasi polynomial above by considering different values of $m$ and $k^{\prime}$. Thus, it can be deduced that the number of voting situations corresponding to each of the strict preferences represented by the cases 26 and 27 in Table 1 is given by $F_{1}\left(m, k^{\prime}\right)$ if both $m$ and $k^{\prime}$ are odd (or even) and by $F_{2}\left(m, k^{\prime}\right)$ if one of the parameters $\left(m\right.$ or $\left.k^{\prime}\right)$ is odd and the other one is even such that:

$$
\begin{aligned}
F_{1}\left(m, k^{\prime}\right)= & \frac{1}{3840}\left(\left(m-3 k^{\prime}-4\right)\left(m-3 k^{\prime}\right)\left(m-3 k^{\prime}+4\right)\right. \\
& \left.\left(m-3 k^{\prime}-2\right)\left(m-3 k^{\prime}+2\right)\right) . \\
F_{2}\left(m, k^{\prime}\right)= & \frac{1}{3840}\left(\left(m-3 k^{\prime}+3\right)\left(m-3 k^{\prime}+7\right)\left(m-3 k^{\prime}+1\right)\right. \\
& \left.\left(m-3 k^{\prime}+5\right)\left(m-3 k^{\prime}-1\right)\right) .
\end{aligned}
$$

As a consequence of the above number of voting situations and taking into account the symmetry of the strict preferences of the cases 26 and 27 in Table 1 and the total number of voting situations $\psi(m)$ in $(5)$, we introduce the probabilities of having triple-acyclic strict preferences under $M_{k^{\prime}}^{L}$ majorities in the following result.

Proposition 1 Consider a three candidate election with $m$ voters under $M_{k^{\prime}}^{L}$ majority rules where each individual vote consists of a linear preference ordering on the candidates. Assuming that all voting situations are equally likely $(I A C)$, if $k^{\prime} \leq(m-3) / 3$, the probability of triple-acyclic strict preference is as follows:

- If both $m$ and $k^{\prime}$ are odd (or even):

$$
1-\frac{2 F_{1}\left(m, k^{\prime}\right)}{\psi(m)} .
$$

- If one of the parameters ( $m$ or $\left.k^{\prime}\right)$ is odd and the other one is even:

$$
1-\frac{2 F_{2}\left(m, k^{\prime}\right)}{\psi(m)}
$$

Computed values of this probability are listed in Table 2 . The values of the difference of votes $k^{\prime}$ correspond to the ones that provide a probability of triple-acyclic strict preferences equal to 1 . As it is previously mentioned, these probabilities indicate that the number of solutions in the systems of inequalities corresponding to the cyclic strict preferences (cases 26 and 27 in Table 1) are equal to zero when $k^{\prime}>(m-3) / 3$.

Some interesting facts could be emphasized from the probabilities in Table 2 .

3 Such simplification is done with Maple software. 
Table 2 Probability of triple-acyclic $P_{k^{\prime}}^{L}$.

\begin{tabular}{||r|rrrrrrr||}
\hline $\begin{array}{r}m \rightarrow \\
k^{\prime} \downarrow\end{array}$ & 3 & 4 & 5 & 10 & 100 & 1,000 & 100,000 \\
\hline 0 & 0.9643 & 1 & 0.9524 & 0.9860 & 0.9462 & 0.9384 & 0.9375 \\
1 & 1 & & 1 & 0.9860 & 0.9462 & 0.9384 & 0.9375 \\
2 & & & & 1 & 0.9605 & 0.9403 & 0.9375 \\
32 & & & & & 1 & 0.9628 & 0.9378 \\
332 & & & & & & 1 & 0.9406 \\
33,332 & & & & & & & 1 \\
\hline
\end{tabular}

First, $M_{0}^{L}$ majority provide a probability of triple-acyclic strict preferences equal to 1 for the case of $m=4$ whereas a difference of votes equal to 1 is necessary in the case of $m=3$ and $m=5$ to achieve such probability. We conjecture that this odd result attends to the fact that the likelihood of having ties is greater in the case of an even number of individuals than in the case of an odd number of individuals when these voters are endowed with linear orderings.

Second, the weight of the difference of votes necessary to achieve a probability of triple-acyclic strict preferences over the total number of votes equal to 1 increases with the number of individuals from $m=10$ to $m=100,000$. In the case of $m=10$ required difference signifies a $20 \%$ of the value of $m$, a $32 \%$ in the case of $m=100$, a $33.2 \%$ in the case of $m=1,000$ and a $33.332 \%$ in the case of $m=100,000$.

Third, the probabilities do not reflect small changes in the magnitude of the thresholds. See for instance, the probabilities attached to the cases $m=10$, $m=100$ and $m=1,000$ for the values of the difference $k^{\prime}$ equal to 0 and 1 and for the case of $m=100,000$ in the case of $k^{\prime}$ equal 0, 1 and 2 .

Finally, triple-acyclic strict preferences under $M_{k^{\prime}}^{L}$ majorities can be guaranteed with a probability of 1 for not too demanding differences of votes.

3.2 Probabilities of transitive strict preferences under majorities based on difference of votes with linear orderings

To study the probability of transitive strict preferences under $M_{k^{\prime}}^{L}$ majorities, we follow the same methodology as the one applied in Subsection 3.1 adding the cases 20,21,22,23,24 and 25 in Table 1 to the outcomes 26 and 27 analyzed in the case of triple-acyclic strict preferences. Once we calculate the probability of non transitive strict preferences collected in the cases from 20 to 27 , we determine the probability of transitive strict preferences as 1 minus the previous probability.

Ordinary preferences collected in cases from 20 to 25 are similar and hence, the number of integer solutions given by Barvinok's algorithm is the same in each of the six systems of inequalities representing these strict preferences. 
For these cases, two validity domains can be distinguished. On the one hand, if $k^{\prime} \leq(m-3) / 3$, the number of voting situations is given by $G_{1}\left(m, k^{\prime}\right)$ if both $m$ and $k^{\prime}$ are odd (or even) and by $G_{2}\left(m, k^{\prime}\right)$ if one of the parameters ( $m$ or $k^{\prime}$ ) is odd and the other one is even such that:

$$
\begin{aligned}
G_{1}\left(m, k^{\prime}\right)= & \frac{1}{1920}\left(k^{\prime}+1\right)\left(121{k^{\prime}}^{4}-116{k^{\prime}}^{3}-200 m k^{\prime 3}+180 m k^{\prime 2}-\right. \\
& 164{k^{\prime}}^{2}+130 m^{2}{k^{\prime}}^{2}+144 k^{\prime}-40 m^{3} k^{\prime}-100 m^{2} k^{\prime}+120 m k^{\prime}- \\
& \left.20 m^{2}-80 m+5 m^{4}+20 m^{3}\right) . \\
G_{2}\left(m, k^{\prime}\right)= & \frac{1}{1920} k^{\prime}\left(121 k^{\prime 4}-200 m k^{\prime 3}-600 k^{\prime 3}+130 m^{2} k^{\prime 2}+780 m k^{\prime 2}+\right. \\
& 910 k^{\prime 2}-40 m^{3} k^{\prime}-360 m^{2} k^{\prime}-840 m k^{\prime}-360 k^{\prime}+5 m^{4}+ \\
& \left.60 m^{3}+210 m^{2}+180 m-71\right) .
\end{aligned}
$$

On the other hand, if $(m-2) / 3 \leq k^{\prime} \leq m-2$, the number of voting situations is given by $G_{3}\left(m, k^{\prime}\right)$ if both $m$ and $k^{\prime}$ are odd (or even) and by $G_{4}\left(m, k^{\prime}\right)$ if one of the parameters $\left(m\right.$ or $\left.k^{\prime}\right)$ is odd and the other one is even such that:

$$
\begin{aligned}
G_{3}\left(m, k^{\prime}\right)= & \frac{1}{3840}\left(\left(m-k^{\prime}-2\right)\left(m-k^{\prime}+4\right)\left(m-k^{\prime}\right)\right. \\
& \left.\left(m-k^{\prime}+6\right)\left(m-k^{\prime}+2\right)\right) . \\
G_{4}\left(m, k^{\prime}\right)= & \frac{1}{3840}\left(\left(m-k^{\prime}+7\right)\left(m-k^{\prime}+3\right)\left(m-k^{\prime}-1\right)\right. \\
& \left.\left(m-k^{\prime}+5\right)\left(m-k^{\prime}+1\right)\right) .
\end{aligned}
$$

Bearing in mind above numbers of voting situations, the results in Proposition 1 and the total number of voting situations $\psi(m)$ in $(5)$, we derive the probability of transitive strict preferences under $M_{k^{\prime}}^{L}$ majorities as follows.

Proposition 2 Consider a three candidate election with $m$ voters under $M_{k^{\prime}}^{L}$ majority rule where each individual vote consists of a linear preference ordering on the candidates. Assuming that all voting situations are equally likely (IAC), the probability of transitive strict preferences is as follows:

1. If $k^{\prime} \leq(m-3) / 3$

- If both $m$ and $k^{\prime}$ are odd (or even):

$$
1-\frac{2 F_{1}\left(m, k^{\prime}\right)+6 G_{1}\left(m, k^{\prime}\right)}{\psi(m)} .
$$

- If one of the parameters ( $m$ or $\left.k^{\prime}\right)$ is odd and the other is even:

$$
1-\frac{2 F_{2}\left(m, k^{\prime}\right)+6 G_{2}\left(m, k^{\prime}\right)}{\psi(m)} .
$$


2. If $(m-2) / 3 \leq k^{\prime} \leq m-2$

- If both $m$ and $\overline{k^{\prime}}$ are odd (or even):

$$
1-\frac{6 G_{3}\left(m, k^{\prime}\right)}{\psi(m)}
$$

- If one of the parameters ( $m$ or $k^{\prime}$ ) is odd and the other is even:

$$
1-\frac{6 G_{4}\left(m, k^{\prime}\right)}{\psi(m)} .
$$

Computed values of this probability are listed in Table 3. Going deeper on them, the weight of the required difference of votes $k^{\prime}$ to guarantee a probability of transitive strict preferences equal to 1 with respect to the total number of individuals increases as the number of individuals does. To illustrate, in the case of $m=3$ the required $k^{\prime}=1$ represents around a $33.33 \%$ of the value of $m$ whereas in the case of $m=100,000$ the required $k^{\prime}$ represents a $99.998 \%$ of the value of $m$. In fact, the required differences are very large for all the considered cases with the exception of $m=3$. Even for $m=4$, the difference signifies a $50 \%$ of the value of $m$. For not too demanding differences, the probabilities increase in the cases of $m=4, m=5$ and $m=10$; this is not the case for $m=100, m=1,000$ and $m=100,000$ where asking reasonable differences of votes decreases the probability of transitive strict preferences.

\begin{tabular}{|c|c|c|c|c|c|c|c|}
\hline $\begin{array}{c}m \rightarrow \\
k^{\prime} \downarrow\end{array}$ & 3 & 4 & 5 & 10 & 100 & 1,000 & 100,000 \\
\hline 0 & 0.9643 & 0.9524 & 0.9524 & 0.9161 & 0.9293 & 0.9366 & 0.9375 \\
\hline 1 & 1 & 0.9524 & 0.9762 & 0.9161 & 0.9293 & 0.9366 & 0.9375 \\
\hline 2 & & 1 & 0.9762 & 0.9580 & 0.9175 & 0.9348 & 0.9375 \\
\hline 3 & & & 1 & 0.9580 & 0.9175 & 0.9348 & 0.9375 \\
\hline 8 & & & & 1 & 0.9088 & 0.9298 & 0.9374 \\
\hline 98 & & & & & 1 & 0.9101 & 0.9366 \\
\hline 998 & & & & & & 1 & 0.9290 \\
\hline 99,998 & & & & & & & 1 \\
\hline
\end{tabular}

Table 3 Probability of transitive $P_{k^{\prime}}^{L}$.

Moreover, as in the case of the probabilities stated in Table 2, small variations in the magnitude of the differences of votes do not change, at least in a significant way, the probabilities. To illustrate, look at the probabilities of $m=100,000$ with differences $k^{\prime}$ equal to $0,1,2$ and 3 .

3.3 Probabilities of transitive weak preferences under majorities based on difference of votes with linear orderings

To derive the probability of transitive weak preferences under $M_{k^{\prime}}^{L}$ majorities, we need to consider, in addition with the cases analyzed in Proposition 2, the 
cases from 14 to 19 in Table 1 . With that, we calculate the probability of non transitive weak preferences and therefore, the probability of transitive weak preferences is determined as 1 minus the probability of non transitive weak preferences.

By symmetry arguments, the weak preferences represented in cases from 14 to 19 are similar and therefore the number of integer solutions of the six systems of inequalities corresponding to such cases is the same. Using again the Barvinok's algorithm, two validity domains can be considered. If $k^{\prime} \leq$ $(m-2) / 3$, the number of voting situations inside each system is given by $H_{1}\left(m, k^{\prime}\right)$ if both $m$ and $k^{\prime}$ are odd (or even), and by $H_{2}\left(m, k^{\prime}\right)$ if one of the parameters $\left(m\right.$ or $\left.k^{\prime}\right)$ is odd and the other one is even such that:

$$
\begin{aligned}
H_{1}\left(m, k^{\prime}\right)= & -\frac{1}{240}\left(( k ^ { \prime } + 1 ) \left(17{k^{\prime}}^{4}-30 m k^{\prime 3}-22{k^{\prime}}^{3}+20 m^{2} k^{2}-\right.\right. \\
& 28{k^{\prime}}^{2}+30 m{k^{\prime}}^{2}+50 m k^{\prime}+48 k^{\prime}-5 m^{3} k^{\prime}-5 m^{2} k^{\prime}- \\
& \left.\left.5 m^{3}-30 m^{2}-40 m\right)\right) . \\
H_{2}\left(m, k^{\prime}\right)= & -\frac{1}{240} k^{\prime}\left(17{k^{\prime}}^{4}-30 m k^{\prime 3}-90{k^{\prime}}^{3}+20 m^{2}{k^{\prime}}^{2}+\right. \\
& 120 m k^{\prime 2}+140{k^{\prime}}^{2}-5 m^{3} k^{\prime}-45 m^{2} k^{\prime}-100 m k^{\prime}- \\
& \left.30 k^{\prime}-37-5 m^{2}-30 m\right) .
\end{aligned}
$$

For the second validity domain, if $(m-1) / 3 \leq k^{\prime} \leq m-1$, this number is given by $H_{3}\left(m, k^{\prime}\right)$ if both $m$ and $k^{\prime}$ are odd (or even) and by $H_{4}\left(m, k^{\prime}\right)$ if one of the parameters $\left(m\right.$ or $\left.k^{\prime}\right)$ is odd and the other one is even such that:

$$
\begin{aligned}
H_{3}\left(m, k^{\prime}\right)= & \frac{1}{3840}\left(\left(m-k^{\prime}+2\right)\left(m-k^{\prime}\right)\left(m-k^{\prime}+4\right)\right. \\
& \left.\left(29{k^{\prime}}^{2}+12 m k^{\prime}+94 k^{\prime}+72-m^{2}+6 m\right)\right) . \\
H_{4}\left(m, k^{\prime}\right)= & \frac{1}{3840}\left(\left(m-k^{\prime}+1\right)\left(m-k^{\prime}+5\right)\left(m-k^{\prime}+3\right)\right. \\
& \left.\left(29{k^{\prime}}^{2}+12 m k^{\prime}+36 k^{\prime}-6 m+7-m^{2}\right)\right) .
\end{aligned}
$$

Taking into consideration the intersections between the different validity domains and using the results in Propositions 1 and 2, the probability of transitive weak preferences is as follows.

Proposition 3 Consider a three candidate election with $m$ voters under $M_{k^{\prime}}^{L}$ majority rule where each individual vote consists of a linear preference ordering on the candidates. Assuming that all voting situations are equally likely (IAC), the probability of transitive weak preferences is as follows:

1. If $k^{\prime} \leq(m-3) / 3$ 
- If both $m$ and $k^{\prime}$ are odd (or even):

$$
1-\frac{2 F_{1}\left(m, k^{\prime}\right)+6 G_{1}\left(m, k^{\prime}\right)+6 H_{1}\left(m, k^{\prime}\right)}{\psi(m)} .
$$

- If one of the parameters ( $m$ or $k^{\prime}$ ) is odd and the other one is even:

$$
1-\frac{2 F_{2}\left(m, k^{\prime}\right)+6 G_{2}\left(m, k^{\prime}\right)+6 H_{2}\left(m, k^{\prime}\right)}{\psi(m)} .
$$

2. If $(m-1) / 3 \leq k^{\prime} \leq m-2$

- If both $m$ and $\bar{k}^{\prime}$ are odd (or even):

$$
1-\frac{6 G_{3}\left(m, k^{\prime}\right)+6 H_{3}\left(m, k^{\prime}\right)}{\psi(m)} .
$$

- If one of the parameters ( $m$ or $k^{\prime}$ ) is odd and the other one is even:

$$
1-\frac{6 G_{4}\left(m, k^{\prime}\right)+6 H_{4}\left(m, k^{\prime}\right)}{\psi(m)} .
$$

3. If $k^{\prime}=(m-2) / 3$

- Either both $m$ and $k^{\prime}$ are odd or both are even:

$$
1-\frac{6 H_{1}\left(m, k^{\prime}\right)+6 G_{3}\left(m, k^{\prime}\right)}{\psi(m)} .
$$

4. If $k^{\prime}=m-1$

- One of the parameters ( $m$ or $k^{\prime}$ ) is odd and the other one is even:

$$
1-\frac{6 H_{4}\left(m, k^{\prime}\right)}{\psi(m)}
$$

Analyzing the probabilities of transitive weak preferences displayed in Table $4, M_{0}^{L}$ majority provides the highest values for the probability of having transitive weak preferences for almost all the considered values of $m$. In fact, any difference of votes can be asked to guarantee a probability value of 1 . Only in the cases of $m=1,000$ and $m=100,000$ the probability arrives to the value of 1.0000 , i.e. the probability approximates to the value of 1 without reaching it ${ }^{4}$. Even so, in both cases the required difference of votes is extremely large. Specifically, it represents a $99.9 \%$ of the value of $m$ in the case of $m=1,000$ and a $99.999 \%$ in the case of $m=100,000$.

Finally, as in the previous cases stated in Tables 2 and 3, the probabilities do not significantly change with small variations of the magnitude of the difference in votes. On this, see for instance the cases of $k^{\prime}$ equal 2 and 3 for $m$ equal 4, 10, 100, 1,000 and 100,000.

4 Notice that this value also appears in Tables $5,7,12,13,14$ and 15 with the same meaning. 
Table 4 Probability of transitive $R_{k^{\prime}}^{L}$.

\begin{tabular}{||r|rrrrrrr}
\hline $\begin{array}{r}m \rightarrow \\
k^{\prime} \downarrow\end{array}$ & 3 & 4 & 5 & 10 & 100 & 1,000 & 100,000 \\
\hline 0 & 0.9643 & 0.7619 & 0.9524 & 0.8462 & 0.9280 & 0.9366 & 0.9375 \\
2 & 0.6786 & 0.7143 & 0.6667 & 0.6703 & 0.9062 & 0.9346 & 0.9375 \\
3 & & 0.7143 & 0.7619 & 0.6703 & 0.9062 & 0.9346 & 0.9375 \\
4 & & & 0.7619 & 0.6503 & 0.8818 & 0.9327 & 0.9375 \\
9 & & & & 0.9101 & 0.8292 & 0.9287 & 0.9374 \\
99 & & & & & 0.9997 & 0.8136 & 0.9366 \\
999 & & & & & & 1.0000 & 0.9276 \\
99,999 & & & & & & & 1.0000
\end{tabular}

4 Probabilities of consistent collective decisions under majorities based on difference of votes with weak orderings

In this section the results about the probabilities of consistent collective decisions under $M_{k^{\prime}}$ majorities are introduced under the IAC assumption. Given that voters could be indifferent between the alternatives, we have to take into account the six linear preference orderings in (4), the six possible orderings that collect the partial indifference and the one that represents the complete indifference among three alternatives. Therefore,

$$
\begin{array}{lll}
x_{1} x_{2} x_{3}\left(m_{1}\right) & x_{1} x_{3} x_{2}\left(m_{2}\right) & x_{2} x_{1} x_{3}\left(m_{3}\right) \\
x_{2} x_{3} x_{1}\left(m_{4}\right) & x_{3} x_{1} x_{2}\left(m_{5}\right) & x_{3} x_{2} x_{1}\left(m_{6}\right) \\
\left\{x_{1} x_{2}\right\} x_{3}\left(m_{7}\right) & \left\{x_{1} x_{3}\right\} x_{2}\left(m_{8}\right) & \left\{x_{2} x_{3}\right\} x_{1}\left(m_{9}\right) \\
x_{1}\left\{x_{2} x_{3}\right\}\left(m_{10}\right) & x_{2}\left\{x_{1} x_{3}\right\}\left(m_{11}\right) & x_{3}\left\{x_{1} x_{2}\right\}\left(m_{12}\right) \\
\left\{x_{1} x_{2} x_{3}\right\}\left(m_{13}\right) & &
\end{array}
$$

where $m_{i}$ represents the number of voters with the associated preference ordering and $\left\{x_{i} x_{j}\right\}$ stands for the indifference between the alternatives $x_{i}$ and $x_{j}$. As the IAC condition is assumed, all possible voting situations $\boldsymbol{m}$ are equally liked to be observed. For $m$ individuals and 3 alternatives, if the indifference between alternatives is allowed, the total number of voting situations $\boldsymbol{m}$ is given by the expression:

$$
\Psi(m)=\frac{(m+1)(m+2) \cdots(m+12)}{12 !} .
$$

Using the same approach applied in Section 3, the probability of consistent outcomes is calculated by means of the computation of the probability of inconsistent outcomes. As there, such probabilities are given by Ehrhart polynomials that provide the number of integer points inside the systems of inequalities that characterize each of the analyzed inconsistent outcomes.

In the framework of the preferences represented in (6), the complexity of the conditions makes impossible the derivation of a general mathematical 
representation as the one provided in Section 3. This is because for each considered consistency condition, the number of validity domains and the length of the polynomials are greater than in the cases of Section 3.

Fortunately, when the number of individuals $m$ and the threshold $k^{\prime}$ are fixed, the probabilities can be calculated for the given number of voting situations $\boldsymbol{m}$.

4.1 Probabilities of triple-acyclic strict preferences under majorities based on difference of votes with weak orderings

In Table 5, the probabilities of triple-acyclic strict preferences under $M_{k^{\prime}}$ majorities are displayed.

The following facts can be pointed our from these results. The probabilities of having triple-acyclic strict preferences when no difference of votes is required, i.e. when $M_{0}$ majority is applied, reach very high values. Specifically, they are located between 0.9571 and 0.9989. For $m=3, m=4$ and $m=5$, the needed difference of votes to achieve a probability value of 1 equals 1 . Therefore, it represents a one third of the value of $m$ in the case of $m=3$, a $25 \%$ in the case of $m=4$ and a $20 \%$ in the case of $m=5$. For the remaining considered values, the weight of the required differences represent around one third of the value of $m$ which means that we can guarantee with a probability of 1 the triple-acyclicity of strict preferences under $M_{k^{\prime}}$ majorities for reasonable values of the difference of votes.

Table 5 Probability of triple-acyclic $P_{k^{\prime}}$.

\begin{tabular}{||r|rrrrrrr||}
\hline $\begin{array}{r}m \rightarrow \\
k^{\prime} \downarrow\end{array}$ & 3 & 4 & 5 & 10 & 100 & 1,000 & 100,000 \\
\hline 0 & 0.9956 & 0.9989 & 0.9929 & 0.9873 & 0.9627 & 0.9577 & 0.9571 \\
1 & 1 & 1 & 1 & 0.9992 & 0.9720 & 0.9589 & 0.9572 \\
2 & & & & 1.0000 & 0.9794 & 0.9601 & 0.9572 \\
3 & & & & 1 & 0.9851 & 0.9613 & 0.9572 \\
33 & & & & & 1 & 0.9852 & 0.9576 \\
333 & & & & & & 1 & 0.9611 \\
33,333 & & & & & & & \\
\hline
\end{tabular}

4.2 Probabilities of transitive strict preferences under majorities based on difference of votes with weak orderings

In Table 6 , the probabilities of transitive strict preferences under $M_{k^{\prime}}$ majorities are presented.

It is remarkable that to reach a probability of transitive strict preferences equal to 1 , the weight of the required difference of votes $k^{\prime}$ with respect to the 
Table 6 Probability of transitive $P_{k^{\prime}}$.

\begin{tabular}{|c|c|c|c|c|c|c|c|}
\hline $\begin{array}{c}m \rightarrow \\
k^{\prime} \downarrow\end{array}$ & 3 & 4 & 5 & 10 & 100 & 1,000 & 100,000 \\
\hline 0 & 0.9692 & 0.9626 & 0.9531 & 0.9422 & 0.9519 & 0.9565 & 0.9571 \\
\hline 1 & 1 & 0.9901 & 0.9884 & 0.9632 & 0.9443 & 0.9554 & 0.9571 \\
\hline 2 & & 1 & 0.9971 & 0.9851 & 0.9399 & 0.9542 & 0.9571 \\
\hline 3 & & & 1 & 0.9942 & 0.9383 & 0.9531 & 0.9571 \\
\hline 8 & & & & 1 & 0.9516 & 0.9484 & 0.9570 \\
\hline 98 & & & & & 1 & 0.9597 & 0.9560 \\
\hline 998 & & & & & & 1 & 0.9471 \\
\hline 99,998 & & & & & & & 1 \\
\hline
\end{tabular}

total number of individuals increases as the number of individuals does. For instance, in the case of $m=4$, it represents a $50 \%$ of the value of $m$ whereas in the case of $m=1,000$ it does a $99.8 \%$ of the value of $m$.

Moreover, the required differences are too demanding for all the cases with the exception of the case of $m=3$ where it represents one third of the value of $m$.

4.3 Probabilities of transitive weak preferences under majorities based on difference of votes with weak orderings

Probabilities of transitive weak preferences under $M_{k^{\prime}}$ majorities defined for weak orderings are displayed in Table 7.

Table 7 Probability of transitive $R_{k^{\prime}}$.

\begin{tabular}{||r|rrrrrrr||}
\hline $\begin{array}{r}m \rightarrow \\
k^{\prime} \downarrow\end{array}$ & 3 & 4 & 5 & 10 & 100 & 1,000 & 100,000 \\
\hline 0 & 0.9801 & 0.8538 & 0.8920 & 0.9072 & 0.9511 & 0.9565 & 0.9571 \\
2 & 0.7890 & 0.7429 & 0.7014 & 0.6980 & 0.9218 & 0.9540 & 0.9571 \\
3 & & 0.8648 & 0.8167 & 0.6814 & 0.9049 & 0.9527 & 0.9571 \\
4 & & & 0.9176 & 0.7234 & 0.8871 & 0.9514 & 0.9571 \\
9 & & & & 0.9919 & 0.7954 & 0.9444 & 0.9570 \\
99 & & & & & 1.0000 & 0.7782 & 0.9559 \\
999 & & & & & & 1.0000 & 0.9436 \\
99,999 & & & & & & & 1.0000 \\
\hline
\end{tabular}

The probability value of 1 is almost achieved for the values of $m$ equal to $100,1,000$ and 100,000. In these cases, the required differences in votes $k^{\prime}$ are so high that signify a $98 \%$ of the value of $m$ in the case of $m=100$, a $99.8 \%$ in the case of $m=1,000$ and a $99.998 \%$ in the case of $m=100,000$. 


\section{Probabilities of consistent collective decisions under majorities based on difference in support}

In this section, we provide the probabilities of reaching consistent collective decisions under $\widetilde{M}_{k}$ majorities for three alternatives.

As long as the intensities of preference between each pair of alternatives can take any value in the continuous interval $[0,1]$, the IAC model can not be applied to provide an a priori probability to each possible voting situation. Therefore the probabilistic analysis carried out in Sections 3 and 4 turns impossible to study the case of $\widetilde{M}_{k}$ majorities.

Consequently, we perform a simulation with the software Matlab to estimate these probabilities as the proportion of the number of consistent outcomes in the simulation over the total number of simulated outcomes. We generate for each of these values 100,000 outcomes to guarantee our results with a confidence level of $99 \%$ and a sampling error of less than a $0.0041 \%^{5}$.

Below, we describe the methodology applied in the simulations to estimate the probability for the considered three types of consistent collective decisions under $\widetilde{M}_{k}$ majorities, i.e. transitive weak preferences, transitive and tripleacyclic strict preferences. We follow that scheme taking into account each type of individual transitive reciprocal relations, i.e. 0.5 -transitive, min-transitive, am-transitive and max-transitive reciprocal preference relations. Notice that the matrix in (1) representing a reciprocal preference relation is determined by the vector composed of the intensities $r_{12}, r_{23}$ and $r_{13}$.

1. We randomly generate $m$ vectors representing the transitive reciprocal preference relations of the $m$ individuals. Such vectors are built bearing in mind one of the considered transitivity conditions for reciprocal preference relations.

2. We compute the sum of the individuals' intensities of preference over each pair of alternatives through a vector $\boldsymbol{S}=\left(S_{12}, S_{23}, S_{13}\right)$ where $S_{i j}=$ $\sum_{p=1}^{m} r_{i j}^{p}$.

3. Having in mind the conditions in equations (2) and (3) and the value of $k$, the collective decision is evaluated over each pair of alternatives in the vector $\boldsymbol{S}$.

${ }^{5}$ Assuming a proportion of consistent outcomes $P$ on the population of a 50\%, the proportion $p$ in a random sample of size $n \geq 30$ for a confidence level of $99 \%$, diverges from the one of the population in an error of less than $\epsilon$ :

$$
\operatorname{Prob}(|P-p| \leq \epsilon) \geq 0.99 \text {. }
$$

Taken into account that the sample proportion $p$ is distributed as $N(P, \sqrt{P(1-P) / n})$, the sampling error $\epsilon$ is as follows:

$$
\epsilon=z_{\alpha / 2} \sqrt{P(1-P) / n} .
$$

In our case, $n=100,000$ and the corresponding percentile of the normal distribution for a confidence level of $99 \%$ is $z_{\alpha / 2}=2.57$. Thus, $\epsilon \leq 0.00407$. 
4. The collective decision in $\boldsymbol{S}$ is classified following the cases of possible collective outcomes displayed in Table 1 . If it is one of the cases 26 or 27, the strict preference $P_{k}$ is not triple-acyclic. If it is one of the cases from 19 to 27 , the strict preference $P_{k}$ is not transitive. Finally, if it is one of the cases from 14 to 27 , the weak preference $R_{k}$ is not transitive.

5. This four steps are iterated 100,000 times to obtain the number of inconsistent collective decisions. Specifically, the number of simulated outcomes in which the weak preference $R_{k}$ is not transitive, in which the strict preference $P_{k}$ is not transitive and in which $P_{k}$ is not triple-acyclic, respectively.

6 . The number of each considered type of consistent social outcomes is computed as the total number of simulated outcomes, i.e. 100,000, minus the number of inconsistent ones computed in the previous step.

7. Finally, each of the desired probabilities, i.e. the probability of transitive $R_{k}$ and the probability of transitive and triple-acyclic $P_{k}$, is calculated as the number of consistent outcomes over the total number of simulated outcomes.

In the following, the simulated probabilities of consistent collective decisions under $\widetilde{M_{k}}$ majorities are listed in tables.

5.1 Probability of transitive weak preferences under majorities based on difference in support

Tables 8, 9, 10 and 11 provide the probabilities of transitive weak preferences when reciprocal preference relations fulfil 0.5-transitivity, min-transitivity, am-transitivity and max-transitivity, respectively.

Table 8 Probabilities of transitive $R_{k}$ for 0.5 -transitive reciprocal preference relations.

\begin{tabular}{||r|rrrrrrr||}
\hline $\begin{array}{r}m \rightarrow \\
k \downarrow\end{array}$ & 3 & 4 & 5 & 10 & 100 & 1,000 & 100,000 \\
\hline 0 & 0.8835 & 0.8781 & 0.8763 & 0.8751 & 0.8706 & 0.8705 & 0.8728 \\
2.97 & 1 & 0.9836 & 0.9485 & 0.7630 & 0.5927 & 0.7826 & 0.8655 \\
3.81 & & 1 & 0.9964 & 0.9065 & 0.5481 & 0.7544 & 0.8633 \\
4.70 & & & 1 & 0.9748 & 0.5312 & 0.7248 & 0.8609 \\
7.95 & & & & 1 & 0.6729 & 0.6266 & 0.8518 \\
26.40 & & & & & 1 & 0.7012 & 0.7954 \\
84.83 & & & & & & 1 & 0.6156 \\
95.32 & & & & & & & 0.5913 \\
\hline
\end{tabular}

When the required difference in support equals zero, the harder the transitivity condition over the reciprocal preference relations is, the higher the probabilities of having transitive weak preferences are. To illustrate, notice that the probabilities vary in between 0.8705 and 0.8835 considering 0.5 -transitive reciprocal preference relations, between 0.9455 and 0.9563 taking into account 
Table 9 Probabilities of transitive $R_{k}$ for min-transitive reciprocal preference relations.

\begin{tabular}{|c|c|c|c|c|c|c|c|}
\hline $\begin{array}{r}m \rightarrow \\
k \downarrow\end{array}$ & 3 & 4 & 5 & 10 & 100 & 1,000 & 100,000 \\
\hline 0 & 0.9563 & 0.9513 & 0.9505 & 0.9487 & 0.9468 & 0.9476 & 0.9455 \\
\hline 2.96 & 1 & 0.9831 & 0.9528 & 0.8015 & 0.7220 & 0.8814 & 0.9400 \\
\hline 3.84 & & 1 & 0.9966 & 0.9205 & 0.6776 & 0.8576 & 0.9383 \\
\hline 4.80 & & & 1 & 0.9809 & 0.6552 & 0.8307 & 0.9366 \\
\hline 8.32 & & & & 1 & 0.7559 & 0.7432 & 0.9291 \\
\hline 27.23 & & & & & 1 & 0.7702 & 0.8831 \\
\hline 95.32 & & & & & & 1 & 0.7189 \\
\hline
\end{tabular}

Table 10 Probabilities of transitive $R_{k}$ for am-transitive reciprocal preference relations.

\begin{tabular}{|c|c|c|c|c|c|c|c|}
\hline $\begin{array}{r}m \rightarrow \\
k \downarrow\end{array}$ & 3 & 4 & 5 & 10 & 100 & 1,000 & 100,000 \\
\hline 0 & 0.9751 & 0.9710 & 0.9712 & 0.9694 & 0.9676 & 0.9670 & 0.9692 \\
\hline 2.97 & 1 & 0.9812 & 0.9492 & 0.8087 & 0.7738 & 0.9117 & 0.9650 \\
\hline 3.91 & & 1 & 0.9971 & 0.9225 & 0.7295 & 0.8904 & 0.9636 \\
\hline 4.91 & & & 1 & 0.9813 & 0.7043 & 0.8683 & 0.9622 \\
\hline 7.48 & & & & 1 & 0.7370 & 0.8112 & 0.9582 \\
\hline 27.90 & & & & & 1 & 0.7873 & 0.9180 \\
\hline 83.01 & & & & & & 1 & 0.7967 \\
\hline 95.32 & & & & & & & 0.7731 \\
\hline
\end{tabular}

Table 11 Probabilities of transitive $R_{k}$ for $\max$-transitive reciprocal preference relations.

\begin{tabular}{|r|rrrrrrr}
\hline $\begin{array}{r}m \rightarrow \\
k \downarrow\end{array}$ & 3 & 4 & 5 & 10 & 100 & 1,000 & 100,000
\end{tabular}

min-transitive reciprocal preference relations, between 0.9670 and 0.9751 bearing in mind am-transitive reciprocal preference relations and between 0.9726 and 0.9777 in the case of max-transitive reciprocal relations.

In the case of $m=100,000$ again, the stronger the required transitivity condition over the reciprocal preference relations is, the higher the probabilities of having transitive weak preferences are. See for instance, the probability values attached to the difference in support $k=95.32$. In the case of $0.5-$ transitive reciprocal preference relations, the probability reaches a value of 0.5913 , whereas it does a value of 0.7189 in the case of min-transitive reciprocal preference relations. In the case of am-transitive reciprocal preference relations 
the probability achieves a value of 0.7731 while it does a value of 0.7744 in the case of max-transitive reciprocal preference relations.

We have found some unexpected results with respect to the needed thresholds to reach probability values equal to 1 for the considered values of $m$ from 3 to 1,000 . In some of these cases, the thresholds are almost the same ones with independence of the required transitivity condition over the reciprocal preference relations (on this, see the cases of $m=3, m=4$ and $m=5$ ). In the case of $m=100$, the highest thresholds are the ones required when reciprocal preference relations are am-transitive and min-transitive, while the lowest ones are the corresponding thresholds to max-transitive and 0.5-transitive reciprocal preference relations. Finally, in the case of $m=1,000$, the highest threshold needed to achieve a probability value of 1 corresponds to mintransitive reciprocal preference relations. The second highest one corresponds to 0.5 -transitive reciprocal preference relations, the third one to am-transitive reciprocal preference relations and the lowest one to max-transitive reciprocal preference relations. Therefore, in these situations we can not establish a clear relationship between the strength of the transitivity condition fulfilled by the reciprocal preference relations and the size of the needed threshold to achieve a probability value of 1 of having transitive weak preferences.

5.2 Probabilities of transitive strict preferences under majorities based on difference in support

Tables 12, 13, 14 and 15 display the probabilities of transitive strict preferences $P_{k}$ when reciprocal preference relations fulfil 0.5 -transitivity, min-transitivity, am-transitivity and max-transitivity, respectively.

Table 12 Probabilities of transitive $P_{k}$ for 0.5-transitive reciprocal preference relations.

\begin{tabular}{||r|rrrrrrr}
\hline $\begin{array}{r}m \rightarrow \\
k \downarrow\end{array}$ & 3 & 4 & 5 & 10 & 100 & 1,000 & 100,000 \\
\hline \begin{tabular}{r|rrrrrr} 
0 \\
$\| .58$
\end{tabular} & 0.8835 & 0.8781 & 0.8763 & 0.8751 & 0.8706 & 0.8705 & 0.8728 \\
2.97 & 1 & 0.9999 & 0.9995 & 0.9916 & 0.8512 & 0.8287 & 0.8668 \\
3.24 & & 1 & 1.0000 & 0.9970 & 0.8661 & 0.8268 & 0.8659 \\
5.17 & & & 1 & 0.9985 & 0.8759 & 0.8257 & 0.8654 \\
14.51 & & & & 1 & 0.9465 & 0.8267 & 0.8611 \\
47.79 & & & & & 1 & 0.9295 & 0.8440 \\
\hline
\end{tabular}

At a first glance, the probability of transitive strict preferences goes to value of 1 for non zero values of the threshold $k$ for all the considered type of transitive reciprocal preference relations.

The rhythm of the convergence of these probabilities to 1 depends on the number of individuals $m$ because in each of the individual transitivity specifications, it decreases when $m$ increases. 
Table 13 Probabilities of transitive $P_{k}$ for min-transitive reciprocal preference relations.

\begin{tabular}{|c|c|c|c|c|c|c|c|}
\hline $\begin{array}{r}m \rightarrow \\
k \downarrow\end{array}$ & 3 & 4 & 5 & 10 & 100 & 1,000 & 100,000 \\
\hline 0 & 0.9563 & 0.9513 & 0.9505 & 0.9487 & 0.9468 & 0.9476 & 0.9455 \\
\hline 1.96 & 1 & 0.9999 & 0.9997 & 0.9968 & 0.9377 & 0.9271 & 0.9421 \\
\hline 2.49 & & 1 & 1.0000 & 0.9993 & 0.9514 & 0.9255 & 0.9414 \\
\hline 2.61 & & & 1 & 0.9995 & 0.9533 & 0.9254 & 0.9413 \\
\hline 3.47 & & & & 1 & 0.9704 & 0.9248 & 0.9398 \\
\hline 12.51 & & & & & 1 & 0.9793 & 0.9291 \\
\hline 35.24 & & & & & & 1 & 0.9251 \\
\hline 47.79 & & & & & & & 0.9311 \\
\hline
\end{tabular}

Table 14 Probabilities of transitive $P_{k}$ for am-transitive reciprocal preference relations.

\begin{tabular}{||r|rrrrrrr}
\hline $\begin{array}{r}m \rightarrow \\
k \downarrow\end{array}$ & 3 & 4 & 5 & 10 & 100 & 1,000 & 100,000
\end{tabular}

Table 15 Probabilities of transitive $P_{k}$ for max-transitive reciprocal preference relations.

\begin{tabular}{|c|c|c|c|c|c|c|c|}
\hline $\begin{array}{r}m \rightarrow \\
k \downarrow\end{array}$ & 3 & 4 & 5 & 10 & 100 & 1,000 & 100,000 \\
\hline 0 & 0.9777 & 0.9766 & 0.9751 & 0.9741 & 0.9727 & 0.9726 & 0.9744 \\
\hline 1.51 & 1 & 1.0000 & 0.9999 & 0.9981 & 0.9689 & 0.9619 & 0.9727 \\
\hline 1.66 & & 1 & 0.9999 & 0.9989 & 0.9712 & 0.9614 & 0.9725 \\
\hline 1.93 & & & 1 & 0.9996 & 0.9758 & 0.9608 & 0.9723 \\
\hline 2.78 & & & & 1 & 0.9870 & 0.9607 & 0.9715 \\
\hline 9.72 & & & & & 1 & 0.9897 & 0.9662 \\
\hline 26.10 & & & & & & 1 & 0.9626 \\
\hline 47.79 & & & & & & & 0.9690 \\
\hline
\end{tabular}

Notice that the strength of the transitivity condition over the reciprocal preference relations seems to play a role in that convergence. It looks that the more rational the individuals are, the smaller the thresholds different from 0 needed to induce transitive strict preferences are. The unique exception to that behavior is found when $m=100$, where the threshold with attached probability value of 1 is slightly lower in the case of am-transitive reciprocal preference relations (see Table 14) than in the case of max-transitive ones (see Table 15). 
To illustrate these general facts, we focus on the required thresholds for $m=3$ and $m=1,000$ in the four cases. In the case of 0.5 -transitive reciprocal relations in Table 12, the required threshold equals 2.58 and consequently represents a $85 \%$ of the value of $m$. In the case of $m=1,000$, the required threshold represents less than a $4.8 \%$ of the value of $m$. In the case of min-transitive reciprocal relations in Table 13, the threshold for reaching a probability of 1 is 1.96 representing less than a $66 \%$ in the case of $m=3$ whereas it symbolizes around a $3.5 \%$ in the case of $m=1,000$. In the case of am-transitive reciprocal relations in Table 14, the thresholds are 1.56 and 33.10 , representing a $52 \%$ and around a $3.3 \%$ of the considered numbers of individuals, respectively. Finally, in the case of max-transitive reciprocal relations in Table 15, the threshold for $m=3$ equals 1.51 and therefore represents around a $50 \%$ of the number of voters and in the case of $m=1,000$ represents around a $2.6 \%$ of the number of voters.

5.3 Probabilities of triple-acyclic strict preferences under majorities based on difference in support

In Tables 16, 17, 18 and 19, we present the results for the simulated probabilities of having triple-acyclic strict preferences when 0.5 -transitive, mintransitive, am-transitive and max-transitive individual preferences are considered.

Table 16 Probabilities of triple-acyclic $P_{k}$ for 0.5 -transitive reciprocal preference relations.

\begin{tabular}{|c|c|c|c|c|c|c|c|}
\hline $\begin{array}{l}m \rightarrow \\
k \downarrow\end{array}$ & 3 & 4 & 5 & 10 & 100 & 1,000 & 100,000 \\
\hline 0 & 0.8835 & 0.8781 & 0.8763 & 0.8751 & 0.8706 & 0.8705 & 0.8728 \\
\hline 1.32 & 1 & 0.9999 & 0.9998 & 0.9967 & 0.9479 & 0.9013 & 0.8763 \\
\hline 1.50 & & 1 & 1.0000 & 0.9983 & 0.9546 & 0.9052 & 0.8768 \\
\hline 1.91 & & & 1 & 0.9998 & 0.9681 & 0.9135 & 0.8778 \\
\hline 2.65 & & & & 1 & 0.9837 & 0.9270 & 0.8794 \\
\hline 8.29 & & & & & 1 & 0.9834 & 0.8926 \\
\hline 26.60 & & & & & & 1 & 0.9291 \\
\hline
\end{tabular}

Conclusions are similar to the case depicted in Subsection 5.2. First, computed probabilities are high and go to 1 for non zero values of $k$ in all considered the cases.

Second, the weight of the needed threshold to reach a probability value of 1 relative to the number of individuals involved in the voting process increases when the value of $m$ does. For example, consider the probabilities for the case of am-transitive reciprocal preference relations in Table 18. There, for $m=3$, the probability achieves the value of 1 for a $k=0.56$ that represents less than a $19 \%$ of the value of $m$. Instead, it represents a a $11.1 \%$ for $m=10$ and less than a $1.2 \%$ for $m=1,000$. 
Table 17 Probabilities of triple-acyclic $P_{k}$ for min-transitive reciprocal preference relations.

\begin{tabular}{||r|rrrrrrr||}
\hline $\begin{array}{r}m \rightarrow \\
k \downarrow\end{array}$ & 3 & 4 & 5 & 10 & 100 & 1,000 & 100,000 \\
\hline 0 & 0.9563 & 0.9513 & 0.9505 & 0.9487 & 0.9468 & 0.9476 & 0.9455 \\
0.67 & 1 & 0.9998 & 0.9997 & 0.9971 & 0.9812 & 0.9583 & 0.9467 \\
0.88 & & 1 & 1.0000 & 0.9993 & 0.9817 & 0.9612 & 0.9471 \\
0.90 & & & 1 & 0.9993 & 0.9906 & 0.9614 & 0.9472 \\
1.40 & & & & 1 & 0.9906 & 0.9675 & 0.9481 \\
4.56 & & & & & 1 & 0.9913 & 0.9540 \\
14.99 & & & & & & 1 & 0.9687 \\
26.60 & & & & & & & 0.9799 \\
\hline
\end{tabular}

Table 18 Probabilities of triple-acyclic $P_{k}$ for am-transitive reciprocal preference relations.

\begin{tabular}{||r|rrrrrrr||}
\hline $\begin{array}{r}m \rightarrow \\
k \downarrow\end{array}$ & 3 & 4 & 5 & 10 & 100 & 1,000 & 100,000 \\
\hline 0 & 0.9751 & 0.9710 & 0.9712 & 0.9694 & 0.9676 & 0.9670 & 0.9692 \\
0.56 & 1 & 1.0000 & 0.9998 & 0.9986 & 0.9854 & 0.9743 & 0.9698 \\
0.67 & & 1 & 1.0000 & 0.9995 & 0.9876 & 0.9758 & 0.9700 \\
0.83 & & & 1 & 0.9999 & 0.9905 & 0.9775 & 0.9702 \\
1.11 & & & & 1 & 0.9941 & 0.9804 & 0.9706 \\
3.34 & & & & & 1 & 0.9936 & 0.9730 \\
11.68 & & & & & & 1 & 0.9811 \\
26.60 & & & & & & & 0.9911 \\
\hline
\end{tabular}

Table 19 Probabilities of triple-acyclic $P_{k}$ for max-transitive reciprocal preference relations.

\begin{tabular}{||r|rrrrrrr||}
\hline $\begin{array}{r}m \rightarrow \\
k \downarrow\end{array}$ & 3 & 4 & 5 & 10 & 100 & 1,000 & 100,000 \\
\hline 0 & 0.9777 & 0.9766 & 0.9751 & 0.9741 & 0.9727 & 0.9726 & 0.9744 \\
0.67 & 1 & 1.0000 & 0.9999 & 0.9998 & 0.9909 & 0.9804 & 0.9753 \\
0.72 & & 1 & 1.0000 & 0.9999 & 0.9918 & 0.9808 & 0.9753 \\
0.75 & & & 1 & 0.9999 & 0.9922 & 0.9811 & 0.9754 \\
0.99 & & & & 1 & 0.9951 & 0.9834 & 0.9758 \\
3.58 & & & & & 1 & 0.9963 & 0.9786 \\
9.95 & & & & & & 1 & 0.9845 \\
26.60 & & & & & & & 0.9943 \\
\hline
\end{tabular}

Third, it seems that the harder the transitivity condition over the reciprocal preference relations is, the lower the weight of threshold required to achieve a probability of triple-acyclic strict preferences of 1 is. To illustrate, look at the weights of the thresholds of support $k$ over the four considered cases when $m=$ 10. In the case of 0.5-transitive reciprocal preference relations (Table 16), the needed threshold represents a $2.26 \%$ of the number of individuals. In the case of min-transitive reciprocal preference relations (Table 17 ), $k$ symbolizes a $1.4 \%$ of $m$. In the case of am-transitive reciprocal preference relations (Table 18) 
the weight of the threshold is a $1.11 \%$ of the value of $m$. Finally, in the case of max-transitive reciprocal preference relations (Table 19), the weight of the threshold represents a $0.99 \%$ of the number of individuals. As in Subsection 5.2 , the case of $m=100$ constitutes an exception to that general behavior. Again, the threshold in the case of am-transitive reciprocal preference relations (see Table 18) is softly lower than the one in the case of max-transitive reciprocal preference relations (see Table 19).

\section{Discussion}

Since now, we have computed the theoretical probabilities of consistent preferences under majorities based on difference of votes defined for both individual linear orderings and weak orderings, and the simulated probabilities of consistent preferences under majorities based on difference in support.

Notice that the results on the probabilities of triple-acyclic strict preferences under $M_{0}^{L}$ majority in Table 2 and under $M_{0}$ majority are consistent with the corresponding ones in Gehrlein (1997) and in Lepelley and Martin (2001).

Focusing on the results for majorities based on difference of votes with linear orderings (Section 3) and with weak orderings (Section 4) we have the following. In the cases of transitive and triple-acyclic strict preferences, the probabilities are higher considering weak than linear orderings (see Tables 2, 3, 5 and 6 ). In the case of transitive weak preferences, the same is true when $k^{\prime}$ equals 1 and 2 and, with the exception of the case in which $m=4$, also when $k^{\prime}=0$.

Looking at the results of Subsection 5.1, notice that the probabilities under $\widetilde{M}_{0}$ remains the same for each type of transitive reciprocal preference relation with independence of the type of consistency condition required to the collective preference. That counterintuitive result contrasts with the remaining ones in which the probability increases together with the increase of the number of social outcomes considered as consistent. That oddity dues to the following. Taking into account expression (3) the absolute value of the difference between the sum of the intensities of preference $r_{i j}^{p}$ and the sum of the intensities $r_{i j}^{p}$ has to be null to the indifference between alternatives to be declared. As far as Matlab generates random numbers with 15 decimal positions, the indifference between alternatives is almost impossible.

Recently some analytical studies about the consistency of majorities based on difference in support have been developed. These theoretical results rely on the needed threshold to ensure transitive and triple-acyclic strict preferences for different types of transitive reciprocal preference relations.

On the one hand, the case of transitive strict preferences is studied in Llamazares et al (2013). The results can be summarized as follows:

1. The transitivity of the strict preference can not be ensured for any threshold of support $k$ less than $m-1$. 
2. The transitivity of the strict preference can not be ensured for any threshold of support $k$ less than $m$ if the reciprocal preference relations are less demanding than am-transitive ones.

3. The strict preference is transitive for any threshold of support such that $k \in$ $[m-1, m)$ if the reciprocal preference relations are at least am-transitive ones.

On the other hand, the case of triple-acyclic strict preferences is analyzed in Llamazares and Pérez-Asurmendi (2013) with the following results:

1. The triple-acyclicity of the strict preference, in the case of 0.5 -transitive reciprocal preference relations, can be guaranteed if the threshold of support $k$ is located in $[\lfloor 2 m / 3\rfloor, m)$ where $\lfloor a\rfloor$ stands for the integer part of $a$.

2. The triple-acyclicity of the strict preference, in the case of min-transitive and max-transitive reciprocal preference relations, can be guaranteed if the threshold of support $k$ belongs to $[m / 3, m)$.

3. In the case of max-transitive reciprocal preference relations, it conjectures that strict preference relations are triple-acyclic if the threshold $k$ belongs to $[\lfloor 2 m / 3\rfloor / 2, m)$.

The probabilistic results setting here complement the above theoretical ones by the following reasons. First, thresholds with associated probabilities of consistent strict preferences equal to 1 are found for all the considered types of transitive reciprocal preference relations. Second, reasonable thresholds are required to certify the consistency of the strict preference with a probability value of 1 in those cases where theoretical results asked a very high threshold to guarantee such consistency. Third, the conjecture about the needed thresholds in the case of max-transitive reciprocal preference relations seems to be true.

Specifically, in the case of transitive strict preferences with 0.5 -transitive and min-transitive reciprocal preference relations, the probabilities achieve the value of 1 for the considered values of $m$ (see Tables 12 and 13, respectively) whereas as it is said before, the theoretical result asserts that no threshold guarantees the transitivity of the strict preference for such types of reciprocal preference relations.

In the cases of am-transitive and max-transitive reciprocal preference relations, the thresholds that provide a probability value of transitive strict preference relations equal to 1 are lower than the ones that guarantee the transitivity of the strict preference in the theoretical framework. To illustrate, assume $m=1,000$. The theoretical result asserts that the threshold $k$ has to belong to $[999,1,000)$. By contrast, a probability value of 1 is achieved with a threshold of 33.10 in the case of am-transitive reciprocal preference relations and of 26.10 in the case of max-transitive reciprocal preference relations (see Tables 14 and 15, respectively).

In the case of triple-acyclic strict preferences, the thresholds to reach a probability value of 1 again are much lower than the ones required in the theoretical setting. For instance, assume $m=5$. The needed thresholds to certify triple-acyclic strict preferences are at least 3 in the case of 0.5 -transitive 
reciprocal preference relations and $5 / 3$ in the cases of min-transitive and maxreciprocal preference relations. Attending to the probabilistic analysis, the needed thresholds to achieve a probability value of 1 are 1.91 in the case of 0.5 -transitive reciprocal preference relations (see Table 16), 0.90 in the case of min-transitive reciprocal preference relations (see Table 17) and 0.75 in the case of max-transitive reciprocal preference relations (see Table 19).

Attending to the results under the probabilistic approach, the conjecture in Llamazares and Pérez-Asurmendi (2013) seems to be true. In Table 20, we provide some other examples that support such idea. For instance, look at the case of $m=100$. The probability of triple-acyclic strict preference relations equals 1 for $k=\lfloor(2 \cdot 100) / 3\rfloor / 2=33$.

Table 20 Probabilities of triple-acyclic $P_{k}$ in the case of max-transitive reciprocal preference relations with $k=[\lfloor 2 m / 3\rfloor / 2, m)$.

\begin{tabular}{|c|c|c|c|c|c|c|c|c|c|c|c|c|}
\hline$m$ & 3 & 4 & 5 & 6 & 7 & 8 & 48 & 49 & 50 & 99 & 100 & 101 \\
\hline$k$ & 1 & 1 & 1.5 & 2 & 2 & 2.5 & 16 & 16 & 16.5 & 33 & 33 & 33.5 \\
\hline Probability & 1 & 1 & 1 & 1 & 1 & 1 & 1 & 1 & 1 & 1 & 1 & 1 \\
\hline
\end{tabular}

We conclude with a comment about the comparison among the probabilities of consistent preferences under the considered three specifications of the majorities based on differences. Notice that the method used to calculate these probabilities differs from the cases of the two specifications of majorities based on difference of votes to the case of majorities based on difference in support. In the first cases, the probability is calculated by counting the integer points given by systems of inequalities whereas in the second case the probability is simulated by Montecarlo techniques. Accordingly, we can not make any quantitative comparison between the results obtained in the first two cases and in the third one.

Acknowledgements The authors are grateful to Ahmad Fliti, José Luis García-Lapresta, Bonifacio Llamazares, Ana Pérez Espartero and Rachid Seghir for their valuable suggestions and comments. This work is partially supported by the Spanish Ministry of Economy and Competitiveness (Projects ECO2012-32178 and ECO2012-34202).

\section{References}

Balasko Y, Crès H (1997) The probability of Condorcet cycles and super majority rules. J Econ Theory 75:237-270

Campbell CD, Tullock G (1965) A measure of the importance of cyclical majorities. Econ J 75(300):853-857

Cervone DP, Gehrlein WV, Zwicker WS (2005) Which scoring rule maximizes Condorcet efficiency under IAC? Theor Decis 58(2):145-185

Condorcet Md (1785) Essai sur l'Application de l'Analyse à la Probabilité des Décisions Rendues à la Pluralité des Voix. Imprimerie Royale, Paris 
Dasgupta M, Deb R (1996) Transitivity and fuzzy preferences. Soc Choice Welfare 13(3):305318

DeMeyer F, Plott C (1970) The probability of a cyclical majority. Econometrica 38(2):345354

Dubois D, Prade H (1980) Fuzzy Sets and Systems: Theory and Applications. Mathematics in Science and Engineering, Academic Press, New York

Fishburn PC, Gehrlein WV (1980) The Paradox of Voting: Effects of individual indifference and intransitivity. J Public Econ 14(1):83-94

García-Lapresta JL, Llamazares B (2001) Majority decisions based on difference of votes. J Math Econ 35(3):463-481

García-Lapresta JL, Llamazares B (2010) Preference intensities and majority decisions based on difference of support between alternatives. Group Decis Negot 19(6):527-542

García-Lapresta JL, Meneses LC (2005) Individual-valued preferences and their aggregation: Consistency analysis in a real case. Fuzzy Sets Syst 151(2):269-284

Gehrlein WV (1983) Condorcet's Paradox. Theor Decis 15(2):161-197

Gehrlein WV (1997) Condorcet Paradox and the Condorcet efficiency of voting rules. Math Jpn 40:173-199

Gehrlein WV, Fishburn PC (1976) Condorcet's Paradox and anonymous preference profiles. Public Choice 26:1-18

Gehrlein WV, Fishburn PC (1980) Robustness of positional scoring over subsets of alternatives. Appl Math Optim 6:241-255

Gehrlein WV, Fishburn PC (1981) Constant scoring rules for choosing one among many alternatives. Qual Quant 15:203-210

Gehrlein WV, Fishburn PC (1983) Scoring rule sensitivity to weight selection. Public Choice 40:249-261

Houy N (2007) Some further characterizations for the forgotten voting rules. Math Soc Sci 53(1):111-121

Jones B, Radcliff B, Taber C, Timpone R (1995) Condorcet winners and the Paradox of Voting: Probability calculations for weak preference orders. Am Polit Sci Rev 89(1):133144

Klahr D (1966) A computer simulation of the Paradox of Voting. Am Polit Sci Rev 60(2):384390

Lepelley D, Louichi A, Smaoui H (2008) On Ehrhart polynomials and probability calculations in voting theory. Soc Choice Welfare 30:363-383

Lepelley D, Martin M (2001) Condorcet's Paradox for weak preference orderings. Eur J Polit Econ 17:163-177

Llamazares B (2006) The forgotten decision rules: Majority rules based on difference of votes. Math Soc Sci 51(3):311-326

Llamazares B, Pérez-Asurmendi P (2013) Triple-acyclicity in majorities based on difference in support, URL http://mpra.ub.uni-muenchen.de/52218/, Working Paper

Llamazares B, Pérez-Asurmendi P, García-Lapresta JL (2013) Collective transitivity in majorities based on difference in support. Fuzzy Sets Syst 216:3-15

Tovey CA (1997) Probabilities of preferences and cycles with super majority rules. J Econ Theory 75:271-279

Verdoolaege S, Seghir R, Beyls K, et al (2004) Analytical computation of Ehrhart polynomials: Enabling more compiler analysis and optimizations. In: Proceedings of International Conference on Compilers, Architecture, and Synthesis for Embedded Systems, Washington D.C., USA, pp 248-258

Wilson MC, Pritchard G (2007) Probability calculations under the IAC hypothesis. Math Soc Sci 54:244-256

Zadeh LA (1971) Similarity relations and fuzzy orderings. Inform Sci 3(2):177-200 